\title{
IMMUNOFLUORESCENT ASSAY FOR SPECIES DIFFERENTIATION OF INTESTINAL MICROSPORIDIOSIS IN IMMUNOCOMPETENT PATIENTS
}

By

\author{
MONA MOHAMED TOLBA*, AMEL YOUSSEF SHEHAB, \\ ESRAA ABDEL-HAMID MONEER and SAFIA SALEH KHALIL. \\ Department of Parasitology, Medical Research Institute, Alexandria University, \\ Egypt (Correspondence: mona.tolba@alexu.edu.eg)
}

\begin{abstract}
Microsporidia constitutes a diverse group of obligate intracellular pathogens that infect a wide range of vertebrate and invertebrate hosts. It has been originally recognized as opportunistic pathogens of immunocompromised patients. Two species of microsporidia, Encephalitozoon bieneusi and Enterocytozoon intestinalis, are known to infect mainly the gastrointestinal tract of humans. The study was based on the examination of stool samples collected from 100 immunocompetent patients complaining of GIT disturbances attending the Parasitology Department laboratory in the Medical Research Institute, Alexandria, Egypt. Freshly collected stool specimens were examined by modified Trichrome stain MTS (Kokoskin hot method) and immunofluorescent monoclonal antibodies (IFA-MAbs).

The results showed that Using MTS, microsporidial spores were detected in $36 \%$ of the stool samples examined. Using IFA-MAbs, 60 samples were positive. Microsporidial spores appeared as bright apple green small oval spores with or without vacoule. The agreement between MTS and IFA-MAbs in diagnosing microsporidal infections was moderate. IFA-MAbs differentiated microsporidial infection as $67 \%$ E. intestinalis, $6 \%$ E. bieneusi and mixed infections in $27 \%$ of positive cases.

Keywords: microsporidia, diagnosis, modified trichrome, immunofluorescence

\section{Introduction}

Microsporidia spp. are obligate intracellular parasites which are very minute with sizes ranging from 1 to $10 \mu \mathrm{m}$, spore-forming parasites infecting mostly all living organisms, especially insects, fish, and mammals (Weber et al, 1994). Microsporidia have attracted considerable attention because they infect a wide range of hosts, from invertebrates to vertebrates, and cause serious human diseases and major economic losses in the livestock industry (Li et al, 2014). They are now being increasingly recognized as important opportunistic pathogens in human immunodeficiency virus (HIV) infection. It was described in immunocompetent hosts (Wittner et al, 1993; Sandfort et al, 1994). More than 1300 microsporidia species were identified and divided into about 150 genera (Keeling and Fast, 2002). About 14 microsporidia species infect humans (Didier and Weiss, 2011).

In Egypt, they were increasingly recognized as human pathogens in AIDS and im-

munocompromised patients, mainly associated with life-threatening chronic diarrhea and systemic disease (Abdel-Hamid et al, 2015). Elnadi et al. (2015) Sohag Governorate reported microsporidia in 3\% of diabetic patients. Al-Herrawy and Gad (2016) Giza Governorate reported animals infected with $E$. bieneusi in a higher percentage than $E$. intestinalis and concluded that domestic animals might play a role in environmental dissemination of intestinal microsporidiosis.

Diseases caused by different microsporidia that infect humans include diarrhea, kerato-conjunctivitis, hepatitis, myositis, urogenital infection, ascites, cholangitis and disseminated disease (Weber et al, 1994). The epidemiology knowledge of microsporidiosis in humans was limited, but as with cryptosporidiosis, microsporidiosis was probably a self-limited or asymptomatic in immunocompetent hosts. High antibodies prevalence to microsporidia (as Encephalitozoon) in many serosurveys supported that view (Wittner $e t$ al, 1993; Weber et al, 1994).
\end{abstract}


The commonest microsporidial infection in man is caused by Enterocytozoon bieneusi and/or Encephalitozoon intestinalis. There was a high prevalence of enteric carriage of $E$. bieneus in the immunocompetent persons in tropical countries (Thellier et al, 2004). This parasite naturally persists in the human population (Hamamci et al, 2015).

Diagnosis is routinely based on the direct demonstration of microsporidian spores in stool samples which requires a high level of expertise in order to be reliable. Microsporidian spores are difficult to detect because of their small size and their staining characteristics, which are similar to those of enteric bacteria and fungi (Chioralia et al, 1998).

During the last decade diagnostic procedures to identify microsporidia in humans were improved remarkably and today several methods for detection and species differentiation of microsporidia are available: transmission electron microscopy, light microscopy (Cytologic diagnosis and stool examinations), histologic diagnosis, cell culture and animal models (Visvesvara et al, 1999; Garcia, 2016).

Antigen-based methods were also used: Immunofluorescent antibody tests with the monoclonal antibodies or polyclonal antibodies (Accoceberry et al, 1999; Thellier et $a l$, 2004). Molecular-based methods like PCR although expensive and time consuming are more accurate and conclusive for detection and species differentiation of microspsoridia (Franzen and Müller, 1999; Kaushik et al, 2017).

Differentiation between the two commonest intestinal microsporidia is required for an adequate therapy management. $E$. intestinalis infections are treated with albendazole, while fumagillin has been shown to be effective for eradicating E. bieneusi. Thus, species identification is important for defining the appropriate treatment which has been possible only using transmission electron microscopy or PCR (Cisse et al, 2002; Thellier et al, 2004).
The study aimed to evaluate stained stool examination and immunofluorescence detection technique in diagnosing microsporidiosis in diarrheic patients

\section{Material and Methods}

Stool samples were collected from 100 diarrheic immunocompetent patients from different areas of Alexandria Governorate attending the Parasitology Department Laboratory, the Medical Research Institute. Each stool sample was subjected to the following:

Microscopic examination: Concentration was performed using the formol-ethyl acetate concentration technique and the sediment was examined for ova and parasites.

Staining technique using: Modified trichrome stain for detection of Microsporidia.

Immunofluorescence detection technique: All stool samples (unpreserved fresh or frozen) were tested by the indirect immunofluorescent antibody assay (IFA) using monoclonal antibodies. Anti-microsporidia Kit, (Bordier Affinity Products SA, Switzerland) was purchased for the detection of microsporidia and differentiation between E. bieneusi and $E$. intestinalis infections.

Prepared fecal sample suspensions were added on 12-well slides, left to dry then different buffers and reagents with monoclonal antibodies for E. bieneusi and E. intestinalis were added according to the manufacturer instructions. (Fig. 1)

Slides were examined under the fluorescence microscope equipped with the appropriate filter for fluorescein and an oil immersion (X 1000).

\section{Results}

The present work enrolled 100 diarrheic patients attending the Parasitology Department in the Medical Research Institute. All collected stool samples were subjected to different diagnostic methods and evaluated.

Formalin ethyl acetate concentration technique (FEAC): The overall percentage of intestinal parasites diagnosed by formalin ethyl acetate was $38 \%$. G. lamblia showed the highest percentage of infection (19\%). 
Modified trichrome stain (MTS): Using MTS, microsporidial spores were detected in 36 out of 100 diarrheic stool samples (36\%). The stained spores appeared as small refractile ovoid or rounded pink bodies with polar bodies at one end and a vacuole at the other. Additional fecal elements, such as yeast, bacteria and the background debris, stained faint pink, sometimes red (Fig. 2).

Immunofluorescent antibody assay (IFAMAbs): Using IFA-MAbs method, 60 out of $100(60 \%)$ fecal specimens examined were positive for microsporidia. Microsporidial spores appeared as bright apple green oval halos, with black background. The monoclonal antibodies react exclusively with the spore walls of microsporidia. Both E. bieneusi spores and E. intestinalis spores were surface labeled with a marked peripheral fluorescence. Yeast cells and bacterial spores exhibited dull fluorescence, yet, could be

Table 1: Agreement between modified trichrome stain (MTS) and immunoflourescent Ab assay (IFA-MAbs) in diagnosis:

\begin{tabular}{|l|c|c|c|c|}
\hline \multirow{2}{*}{ Agreement } & \multicolumn{2}{|c|}{ IFA-MAbs } & \multirow{2}{*}{ Total } \\
\cline { 3 - 5 } & Negative & Positive & \\
\hline \multirow{2}{*}{ Modified Trichrome stain (MTS) } & $-\mathrm{ve}$ & 38 & 26 & 64 \\
\cline { 2 - 5 } & $+\mathrm{ve}$ & 2 & 34 & 36 \\
\hline \multicolumn{2}{|l|}{ Total } & 40 & 60 & 100 \\
\hline
\end{tabular}

\section{Discussion}

Though the pathogenic importance of microsporidia in the population at large is still to be determined, yet, they are increasingly recognized as emerging infectious pathogens causing various diseases in both immunocompetent and immunocompromised patients (Didier and Weiss, 2006).

Chronic diarrhea is the commonest symptom of microsporidial infection especially in immunocompromised patients causing malabsorption and wasting (Kotler and Orenstein, 1998). Many physicians are unaware of microsporidial infection as a cause of unexplained diarrhea (Sak et al, 2011). Rapid and practical means for diagnosis of intestinal microsporidial infection are still lacking in many laboratories. Thus, increasing the awareness for diagnosing microsporidia is of great importance as the organisms are very small and difficult to distinguish from bacte- easily distinguished from the microsporidial spores by bright fluorescence (Figs. 4 \&5).

Regarding the different microsporidia species diagnosed by IFA MAbs, out of 60 specimens diagnosed positive for microsporidial spores: 40 were E. intestinalis (67\%), 4 E. bieneusi $(6 \%) \& 19$ mixed infections $(27 \%)$.

Comparing the results of the 100 diarrheic cases examined both by modified trichrome stain (MTS) and immunoflourescent Ab assay (IFAMAbs), it was revealed that 34 cases were positive by both techniques (concordant positive results). By analysis of the discordant results, 26 cases positive by immunoflourescent $\mathrm{Ab}$ assay were missed by MTS, and two cases diagnosed positive by (MTS) were negative by (IFA-MAb). Statistical analysis showed a Kappa index of 0.47 indicating moderate agreement between MTS and IFA-MAbs in diagnosing microsporidal infections. (Tab.1; Fig 5) ria and small yeasts, thus cannot be identified by routine examination of stool (Garcia 2002).

In the present study, diagnosis of microsporidiosis was based on the detection of microsporidial spores in stool specimens by light microscopy using special stains. A total of 36 out of 100 (36\%) diarrheic stool samples were positive for microsporidia using the modified trichrome stain (Kokoskin hot method).

In the present study, immunofluorescence antibody assay with monoclonal antibodies is highly sensitive and specific for identifying microsporidia species (Accoceberry et al, 1999; Thellier et al, 2004). The monoclonal antibody offers a new vehicle for epidemiological studies geared to determining accurate prevalence of microsporidia. Fluorescence microscopic examination requires a well-trained person to view the preparation 
objectively and requires skilled interpretation which includes some amount of subjectivity (Enriquez, 1997).

In the present study, IFA-MAbs detected microsporidian spores in 60 samples $(60 \%)$ appearing as bright green oval bodies against a black background. This suggested that microsporidia are becoming a highly prevalent intestinal parasite that needs more consideration as an etiological agent for diarrhea in immunocompetent patients.

Several factors explained the lower prevalence of the MTS examination of microsporidian spores as compared to the IFA-MAbs. Regular microscopy is quite difficult, relatively time consuming and highly dependent on the expertise of the examiner. On the other hand, IFA assay overcomes difficulties by being specific for microsporidia only and not staining yeast cells, bacteria, and other protozoa (Thellier et al, 2004).

Hamamci et al. (2015) found that IFAMAbs, modified trichrome, acid fast trichrome and Calcofluor staining methods were performed on 50 stool samples and evaluated. The percentages of microsporidia were as follows; $66 \%(\mathrm{n}=33)$ with IFA-MAbs, $34 \%(\mathrm{n}=17)$ with modified trichrome staining, $24 \%(n=12)$ with acid fast trichrome staining and $42 \%(n=21)$ with Calcofluor staining method. They concluded that the use of IFA-MAbs along with the conventional staining methods in diagnosis of microsporidia will increase the sensitivity.

Identification of microsporidia species is a must for prompt and proper pharmacological therapy to reduce the risk of progression to disseminated infection with a fatal outcome especially in immunocompromised patients. Infections caused by E. intestinalis are treated with albendazole, while fumagillin has been shown to be effective for eradicating $E$. bieneusi (Anane and Attouchi, 2010). Regular microscopy does not allow identification of microsporidia to the species level. The transmission electron microscopy (TEM) remains the gold standard necessary for the identification of the microsporidian species.
However, this technique is expensive, time consuming, and not feasible for routine diagnosis. Moreover, the TEM may not be sensitive enough to detect a small number of microsporidial spores in feces (Garcia, 2016). Therefore, it was beneficial to develop specific IFA-MAbs that can be used for sensitive diagnosis as well as species identification of microsporidia. IFA will also make it possible to determine how common different microsporidial species are in the environment, particularly in drinking water, and in other mammalian species (Mo and Drancourt, 2004). On the other hand, the more sensitive and specific assay is the PCR, which is considered the method of choice for species identification; however, it is not routinely used since it is time consuming, labor intensive and expensive (Espern et al, 2007, Al-Mekhlafi et al, 2011)

The decision of selecting a particular diagnostic technique and approach for incorporation into the work flow in any Parasitology laboratory is based on a number of factors including: clinical significance, cost-effectiveness, work capacity, relative ease, availability of equipment, and finally the ability with which a new procedure fits into the routine laboratory work flow (Garcia, 2016).

\section{Conclusion}

E. intestinalis species was the commonest microsporidia causing intestinal infections in the immunocompetent patients. The IFA besides trichrome stain were recommended for detection of microsporidian spores in stool as being a reliable and less expensive diagnostic technique compared to PCR.

\section{References}

Abdel-Hamid, MY, Badawy, AF, Ibrahim, A N, 2015: An original simple technique for diagnosis of Microsporidia using glycerol jelly direct smear. Parasitol. Res. 114, 2:453-6.

Accoceberry, I, Thellier, M, Desportes-Liva ge, I, Achbarou, A, Biligui, S, et al, 1999: Production of monoclonal antibodies directed against the microsporidium Enterocytozoon bieneusi. J. Clin. Microbiol. 37:4107-12

Al-Herrawy, AZ, Gad, MA, 2016: Microsporidial spores in fecal samples of some domesticat- 
ed animals living in Giza, Egypt. Iran. J. Parasitol. 11, 2:195-203.

Al-Mekhlafi, MA, Fatmah, MS, Anisah, N, Azlin, M, Al-Mekhlafi, HM, Norhayati, M, 2011: Species identification of intestinal microsporidia using immunofluorescence antibody assays. Southeast Asian J. Trop. Med. Publ. Hlth. 42, 1:S19-22

Chioralia, G, Trammer, T, Kampen, H, Seitz, HM, 1998: Relevant criteria for detecting microsporidia in stool specimens. J. Clin. Microbiol. 36, 8:2279-83.

Cisse, OA, Ouattara, A, Thellier, M, Accoceberry, I, Biligui, S, et al, 2002: Evaluation of an immunofluorescent-antibody test using monoclonal antibodies directed against Enterocytozoon bienusi and Encephalitozoon intestinalis for diagnosis of intestinal microsporidiosis in Bamako (Mali). J. Clin. Microbiol. 40:1715-8

Didier, ES, 2005: Microsporidiosis: an emerging and opportunistic infection in humans and animals. Acta Trop. 94:61-76

Didier, ES, Weiss, LM, 2006: Microsporidiosis: Current status. Curr. Opin. Infect. Dis. 19, 5: 485-92

Didier, ES, Weiss, LM, 2011: Microsporidiosis: Not just in AIDS patients. Curr. Opin. Infect. Dis. 24, 5:490-5.

Elnadi, NA, Hassanien, HA, Ahmad, AM, Abd-Ellah, AK, 2015: Intestinal parasites in diabetic patients in Sohag University Hospitals, Egypt. J. Egypt. Soc. Parasitol. 45, 2:443-9.

Enriquez, FJ, Ditrich, O, Palting, JD, Smith, K, 1997: Simple diagnosis of Encephalitozoon sp. microsporidial infections by using a pan-specific anti-exospore monoclonal antibody. J. Clin. Microbiol. 35, 3:724-9

Franzen, C, Müller, A, 1999: Molecular techniques for detection, species differentiation, and phylogenetic analysis of microsporidia. Clin. Microbiol. Rev. 12:243-85

Garcia, LS, 2002: Laboratory identification of the microsporidia. J. Clin. Microbiol. 40:1892901

Garcia, LS, 2016: Diagnostic Medical Parasitology. ASM Press, Washington DC, USA.

Hamamcı, B, Çetinkaya, Ü, Berk, V, Kaynar, L, Kuk, S, 2015: Prevalence of E. intestinalis and $E$. bieneusi in cancer patients under chemotherapy. Mikrobiyol. Bul. 49, 1:105-13.
Kaushik, S, Saha, R, Das, S, Ramachandran, VG, Goel, A, 2017: Pragmatic combination of available diagnostic tools for optimal detection of intestinal Microsporidia. Adv. Exp. Med. Biol. https://doi.org/10.1007/5584.

Keeling, PJ, Fast, NM, 2002: Microsporidia: biology and evolution of highly reduced intracellular parasites. Ann. Rev. Microbiol. 56:93-116. Kotler, DP, Orenstein, JM, 1998: Clinical syndromes associated with microsporidiosis. Adv. Parasitol. 40:321-49.

Li, Z, Hao, Y, Wang, L, Xiang, H, Zhou, Z, 2014: Genome-wide identification and comprehensive analyses of the kinomes in four pathogenic microsporidia species. PLoS One. Dec 30; 9 (12):e115890.

Mo, L, Drancourt, M, 2004: Monoclonal antibodies for specific detection of E. cuniculi. Clin. Diagn. Lab. Immunol. 11, 6:1060-3.

Sak, B, Brady, D, Pelikánová, M, Květoňová, D, Rost, M, et al, 2011: Unapparent microsporidial infection among immunocompetent humans in the Czech Republic. J. Clin. Microbiol 49, 3:1064-70

Sandfort, J, Hannemann, A, Gelderblom, H, Starrk, K, Owen, RL, et al, 1994: Enterocytozoon bieneusi infection in an immunocompetent patient who had acute diarrhea and who was not infected with the human immunodeficiency virus. Clin. Infect. Dis. 19, 3:514-6.

Thellier, MI, Accoceberry, I, Desportes, S, Biligui, E, Bart-Delabesse, C, et al, 2004: Simple species diagnosis of human intestinal microsporidia by an immunofluorescence test using specific monoclonal antibodies (NATO). July 12-15. Ceské Budéjovice.

Visvesvara, GS, Moura, H, Leitch, GJ, Schwartz, DA, 1999: Culture and propagation of microsporidia. In: Wittner, M, Weiss, LM (Eds.). The Microsporidia and Microsporidiosis, American Society for Microbiology, Washington, DC. Weber, R, Bryan, RT, Schwartz, DA, Owen, RL, 1994: Human microsporidial infections. $\mathrm{Cl}-$ in. Microbiol. Rev. 7, 4:426-61.

Wittner, M, Tanowitz, HB, Weiss, LM, 1993: Parasitic infections in AIDS patients: Cryptosporidiosis, isosporiasis, microsporidiosis, cyclosporiasis. Infect. Dis. Clin. North Am. 7, 3:56986. 


\section{Explanation of figures}

Fig 1: Stool samples with both monoclonal Abs on different wells of a glass slide.

Fig. 2: Microsporidial spores by MTS (X1000)

Fig. 3: E. bieneusi by IFA-MAbs (Oil immersion lens, X1000).

Fig. 4: E. intestinalis by IFA-MAbs, (Oil immersion, X1000).

Fig. 5: Moderate agreement $k=.47, \mathrm{p}<.001$
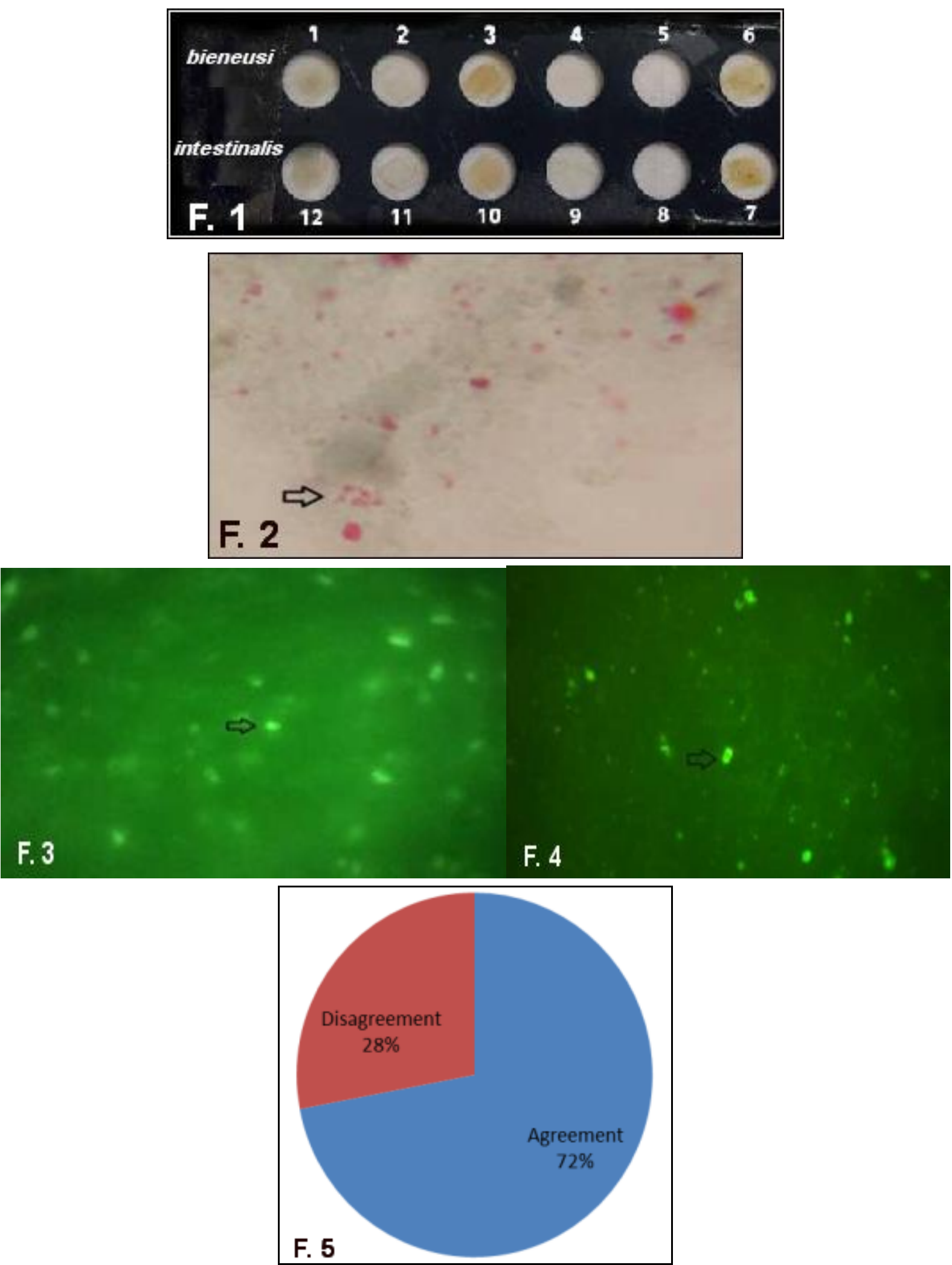